Math. Model. Nat. Phenom.

Vol. 5, No. 4, 2010, pp. 150-157

DOI: $10.1051 / \mathrm{mmnp} / 20105406$

\title{
Weak Asymptotics for Schrödinger Evolution
}

\author{
S. A. Denisov ${ }^{1}$ \\ University of Wisconsin-Madison, Mathematics Department \\ 480 Lincoln Dr., Madison, WI, 53706, USA
}

\begin{abstract}
In this short note, we apply the technique developed in [2] to study the long-time evolution for Schrödinger equation with slowly decaying potential.
\end{abstract}

Key words: Schrödinger evolution, square-summable potential, wave operators AMS subject classification: $35 \mathrm{~J} 10$

Consider

$$
H=-\partial_{x x}^{2}+q, \quad x>0
$$

with Dirichlet boundary condition at zero. If $q=0$, we denote the operator by $H_{0}$. Through the paper, the potentials is real-valued and satisfies the following condition

$$
q(x) \ln (|x|+2) \in L^{2}\left(\mathbb{R}^{+}\right)
$$

We will use the asymptotics of generalized eigenfunctions obtained in [2] to prove existence of modified wave operators. Unfortunately, the limits will be understood in some averaged sense only. In the meantime, the methods are rather robust and can be used for other dispersive equations. For $q \in L^{p}\left(\mathbb{R}^{+}\right), 1 \leq p<2$ the existence of modified wave operators was proved in [1].

We will start with some definitions. Assume that $f(x) \in L^{2}\left(\mathbb{R}^{+}\right)$and take its odd continuation to $\mathbb{R}$. Call it $f_{o}(x)$. Then

$$
e^{i t \partial_{x}^{2}} f_{o} \sim \kappa \frac{e^{i x^{2} /(4 t)}}{\sqrt{t}} \hat{f}_{o}(x /(2 t)) \quad \text { in } L^{2}(\mathbb{R}), \quad t \rightarrow \infty
$$

\footnotetext{
${ }^{1}$ E-mail: denissov@math.wisc.edu
} 
where

$$
\kappa=-\frac{1}{(1+i) \sqrt{2 \pi}}, \quad \hat{f}_{o}(\omega)=\int f_{o}(x) e^{i \omega x} d x
$$

so $\hat{f}_{o}$ denotes the inverse Fourier transform. (In this paper, $f \sim g$ as $t \rightarrow \infty$ if $\|f-g\| \rightarrow 0$ as $t \rightarrow \infty$ in the specified metric). The asymptotics (0.2) is easy to check if $\hat{f}_{o}$ is infinitely smooth and compactly supported away from zero. The general $L^{2}$ case then follows upon making the simple observation that the 1.h.s. and the r.h.s. are unitary in $f$ and then using the approximation argument. Then, by symmetry,

$$
e^{-i H_{0} t} f \sim \kappa \frac{e^{i x^{2} /(4 t)}}{\sqrt{t}} \hat{f}_{o}(x /(2 t)) \chi_{x>0}, \quad \text { in } L^{2}\left(\mathbb{R}^{+}\right), \quad t \rightarrow+\infty
$$

We will need to modify the free evolution. The modification will be made in the physical space as follows

$$
U(t) f=\kappa \frac{e^{i x^{2} /(4 t)}}{\sqrt{t}} \hat{f}_{o}(x /(2 t)) \exp \left(-i \frac{t}{x} \int_{0}^{x} q(s) d s\right)
$$

Let $u(x, k)$ be the solution to the Cauchy problem

$$
-u^{\prime \prime}+q u=k^{2} u, \quad u(0, k)=0, \quad u^{\prime}(0, k)=1, \quad E=k^{2}
$$

and the spectral measure

$$
d \rho_{E}=d \rho_{s}(E)+\mu(E) d E
$$

The negative eigenvalues, if there are any, will be denoted by $\left\{-\kappa_{j}^{2}\right\}, j=1,2, \ldots$ We will need the following trivial lemma

Lemma 1. If $f(x)$ is infinitely smooth function with compact support, then

$$
\left|\frac{1}{\sqrt{t}} \int_{x}^{\infty} f\left(s t^{-1}\right) \exp \left(i\left(\frac{s^{2}}{2 t}-s k\right)\right) d s\right| \lesssim \frac{\sqrt{t}}{x-k t+\sqrt{t}}
$$

for any $x>k t$ and for $t>1, k>0$.

Proof. After the change of variables $s=t(k+u)$, we are left with

$$
e^{-i t k^{2} / 2} \int_{x t^{-1}-k}^{\infty} f(k+u) \sqrt{t} e^{i t u^{2} / 2} d u
$$

Integration by parts and the simple estimate

$$
\left|\int_{x}^{\infty} e^{i u^{2}} d u\right| \lesssim \frac{1}{1+x}, \quad x>0
$$

finish the proof.

Below, we will use some notations from [2]. The main result of the paper is 
Theorem 2. For any $f \in L^{2}\left(\mathbb{R}^{+}\right)$, there is $W_{f}$ such that

$$
\frac{1}{T} \int_{0}^{T}\left\|e^{i t H} U(t) f-W_{f}\right\|_{L^{2}\left(\mathbb{R}^{+}\right)}^{2} d t \rightarrow 0, \quad T \rightarrow \infty
$$

The exact expression for $W_{f}(x)$ will be given in the proof.

Thus, for most large $t$, we have $e^{-i t H} W_{f} \sim U(t) f$ in $L^{2}\left(\mathbb{R}^{+}\right)$.

Proof. It is sufficient to show that

$$
\frac{1}{T} \int_{T}^{2 T}\left\|e^{i t H} U(t) f-W_{f}\right\|_{L^{2}\left(\mathbb{R}^{+}\right)}^{2} d t \rightarrow 0, \quad T \rightarrow \infty
$$

(this follows, e.g., from the diadic decomposition argument).

Assume that $f$ is such that $\hat{f}_{o}(k) \chi_{k>0}$ is infinitely smooth with compact support on, say, $[a, b] \subset$ $\mathbb{R}^{+}$. If we prove the convergence in this case, then the standard approximation argument can handle the general situation. Denote the generalized Fourier transform of $U(t) f$ by $\breve{\psi}(t, k)$. For $k \geq 0$,

$$
\breve{\psi}(t, k)=\int_{0}^{\infty} u(x, k)[U(t) f](x) d x
$$

Using the formula (9) from [2], we have

$$
\breve{\psi}(t, k)=-I_{1}+I_{2}
$$

where

$$
\begin{aligned}
I_{1} & =\frac{1}{2 i k} \int_{2 a t}^{2 b t}[U(t) f](x) j_{m}(k, x) e^{-i k x+i \phi(0, k, x)} d x \\
I_{2} & =\frac{1}{2 i k} \int_{2 a t}^{2 b t}[U(t) f](x) \overline{j_{m}}(k, x) e^{i k x-i \phi(0, k, x)} d x
\end{aligned}
$$

Splitting the integration in $I_{1}$ as

$$
\int_{2 a t}^{2 b t}=\int_{2 a t}^{2 k t}+\int_{2 k t}^{2 b t}=J_{1}+J_{2}
$$

we have

$$
\begin{aligned}
& J_{1}=-\frac{\kappa}{2 i k \sqrt{t}} \int_{2 k t}^{2 b t} j_{m}(k, x) \exp \left(i\left(\frac{1}{2 k}-\frac{t}{x}\right) \int_{0}^{x} q(s) d s\right) \\
& \partial_{x}\left(\int_{x}^{2 b t} \hat{f}_{o}(s /(2 t)) \exp \left(i\left(\frac{s^{2}}{4 t}-s k\right)\right) d s\right) d x
\end{aligned}
$$

Integrating by parts,

$$
J_{1}=L_{1}+L_{2}
$$


where

$$
L_{1}=\frac{\kappa}{2 i k} j_{m}(k, 2 k t)\left(\frac{1}{\sqrt{t}} \int_{2 k t}^{2 b t} \hat{f}_{o}(s /(2 t)) \exp \left(i\left(\frac{s^{2}}{4 t}-s k\right)\right) d s\right)
$$

The stationary phase argument gives

$$
\frac{e^{i t k^{2}}}{\sqrt{t}} \int_{2 k t}^{2 b t} \hat{f}_{o}(s /(2 t)) \exp \left(i\left(\frac{s^{2}}{4 t}-s k\right)\right) d s \rightarrow \hat{f}_{o}(k)(\sqrt{\pi / 2}(1+i))
$$

uniformly in $k$ over any compact. So, for any fixed $\delta, M>0$ and interval $I=[\delta, M]$,

$$
\begin{aligned}
\limsup _{T \rightarrow \infty} \frac{1}{T} \int_{0}^{T} \int_{I}\left|e^{i t k^{2}} L_{1}(k, t)-\frac{\kappa}{2 i k} \hat{f}_{o}(k) j_{m}(k) \chi_{\Theta}(E) \sqrt{\pi / 2}(1+i)\right|^{2} d \rho(E) \lesssim \\
\limsup _{T \rightarrow \infty} \frac{1}{T} \int_{I} d \rho(E) \int_{0}^{T}\left|\hat{f}_{o}(k)\left(j_{m}(k, 2 k t)-j_{m}(k) \chi_{\Theta}(E)\right)\right|^{2} d t
\end{aligned}
$$

where $\Theta=\mathbb{R}^{+} \backslash \Theta_{s}, \Theta_{s}$ is the support of $d \rho_{s}(E)$. In the argument above we also used the uniform bound

$$
\limsup _{T \rightarrow \infty} \frac{1}{T} \int_{0}^{T} d t \int_{I} d \rho(E)\left|j_{m}(k, 2 k t)\right|^{2}<\infty
$$

which follows from the estimate (31) in [2] after change of variables $2 k t=\tau$. Making the change of variables $k t=t_{1}$ in $(0.7)$, we have

$$
\begin{aligned}
\limsup _{T \rightarrow \infty} \frac{1}{T} \int_{I} d \rho(E) \int_{0}^{T} & \left|e^{i t k^{2}} L_{1}(k, t)-\frac{\kappa}{2 i k} \hat{f}_{o}(k) j_{m}(k) \chi_{\Theta}(E) \sqrt{\pi / 2}(1+i)\right|^{2} d t \lesssim \\
& \limsup _{T \rightarrow \infty} \frac{1}{T} \int_{I} d \rho(E) \int_{0}^{C_{1} T}\left|j_{m}\left(k, 2 t_{1}\right)-j_{m}(k) \chi_{\Theta}(E)\right|^{2} d t_{1}
\end{aligned}
$$

The last limit is equal to zero by the theorem 3.1 in [2]. For $L_{2}$, using the formula for derivative of $\partial_{x} j(k, x)([2]$, Lemma 2.1), we get

$$
\begin{aligned}
\left|L_{2}\right| \lesssim & \frac{1}{\sqrt{t}} \int_{2 k t}^{2 b t}\left|j_{m}(k, x)\right|\left(|q(x)|+\frac{t}{x^{2}} \int_{0}^{x}|q(s)| d s\right) \\
& \left(\int_{x}^{\infty} \hat{f}_{o}(s /(2 t)) \exp \left(i\left(\frac{s^{2}}{4 t}-s k\right)\right) d s\right) d x
\end{aligned}
$$

for $k \in I$. By lemma 1 we have an estimate

$$
\left|L_{2}\right| \lesssim \int_{2 k t}^{2 b t}\left|j_{m}(k, x)\right|\left(|q(x)|+\frac{1}{x} \int_{0}^{x}|q(s)| d s\right) \frac{\sqrt{t}}{(x-2 k t)+\sqrt{t}} d x
$$

Making the change of variables $k t=t_{1}$ once again, we have

$$
\frac{1}{T} \int_{T}^{2 T} \int_{I}\left|L_{2}(k, t)\right|^{2} d \rho(E) d t \lesssim M_{1}+M_{2}
$$


where

$$
\begin{aligned}
& M_{1}=\int_{C_{1} T}^{C_{2} T} \int_{I}\left(\int_{2 t_{1}}^{C_{3} t_{1}} \frac{\left|j_{m}(k, x) q(x)\right|}{\left(x-2 t_{1}\right)+\sqrt{T}} d x\right)^{2} d \rho(E) d t_{1} \\
& M_{2}=\int_{C_{1} T}^{C_{2} T} \int_{I}\left(\int_{2 t_{1}}^{C_{3} t_{1}} \frac{\left|j_{m}(k, x) q_{1}(x)\right|}{\left(x-2 t_{1}\right)+\sqrt{T}} d x\right)^{2} d \rho(E) d t_{1}
\end{aligned}
$$

and

$$
q_{1}(x)=\frac{1}{x} \int_{0}^{x}|q(u)| d u
$$

By Young's inequality for convolutions and the following estimate ([2], (31))

$$
\sup _{x} \int_{I}\left|j_{m}(x, k)\right|^{2} d \rho(E)<\infty
$$

we have

$$
\begin{aligned}
M_{1} & \lesssim \int_{I} d \rho(E)\left(\int_{C_{1} T}^{C_{2} T}\left|j_{m}(k, x)\right|^{2} q^{2}(x) d x\right)\left(\int_{0}^{C_{3} T} \frac{d x}{x+\sqrt{T}}\right)^{2} \\
& \lesssim \ln ^{2} T \int_{C_{1} T}^{C_{2} T} q^{2}(x) d x \lesssim \int_{C_{1} T}^{\infty} q^{2}(x) \ln ^{2} x d x \rightarrow 0, \quad T \rightarrow \infty
\end{aligned}
$$

For $M_{2}$, the estimate is similar

$$
\begin{aligned}
& M_{2} \lesssim \ln ^{2} T \int_{C_{1} T}^{C_{2} T} q_{1}^{2}(x) d x \lesssim \frac{\ln ^{2} T}{T}\left(\int_{0}^{C T}|q(u)| d u\right)^{2} \\
& \lesssim \bar{o}(1)+\frac{\ln ^{2} T}{T}\left(\int_{\sqrt{T}}^{C T} q^{2}(x) \ln ^{2}(2+x) d x\right)\left(\int_{\sqrt{T}}^{T} \ln ^{-2}(2+x) d x\right) \rightarrow 0, \quad T \rightarrow \infty
\end{aligned}
$$

The term $J_{2}$ in $(0.5)$ can be handled similarly and we have

$$
\frac{1}{T} \int_{I} d \rho(E) \int_{T}^{2 T}\left|e^{i t k^{2}} J_{1(2)}(k, t)-\frac{\kappa}{2 i k} \hat{f}_{o}(k) j_{m}(k) \chi_{\Theta}(E) \sqrt{\pi / 2}(1+i)\right|^{2} d t \rightarrow 0, \quad T \rightarrow \infty
$$

For $I_{2}$, the analysis is identical with the exception that integration by parts gives

$$
\frac{1}{T} \int_{I} d \rho(E) \int_{T}^{2 T}\left|I_{2}\right|^{2} d t \rightarrow 0
$$

Thus, for any $\delta, M>0$,

$$
\frac{1}{T} \int_{T}^{2 T} \int_{\delta}^{M}\left|\breve{\psi}(t, k)+\frac{\kappa}{2 i k} \hat{f}_{o}(k) j_{m}(k) \chi_{\Theta}(E) \sqrt{2 \pi}(1+i)\right|^{2} d \rho(E) \rightarrow 0
$$


In the statement of the theorem, we can choose $W_{f}(x)$ as the function with generalized Fourier transform equal to

$$
\breve{W}_{f}(E)=-\frac{\kappa}{2 i k} \hat{f}_{o}(k) j_{m}(k) \chi_{\Theta}(E) \sqrt{2 \pi}(1+i)
$$

The function $\hat{f}_{o}(k)$ is infinitely smooth with compact support and $U(t) f$ travels ballistically. We also have $\sup _{t}\|U(t) f\|_{W^{1,2}(\mathbb{R})}<\infty$ and so by taking suitable cutoff near the origin

$$
U(t) f=s_{1}(t)+s_{2}(t)
$$

where $\left\|s_{1}(t)\right\|_{2} \rightarrow 0$ and $s_{2}(t) \in \mathcal{D}\left(|H|^{1 / 2}\right)$ with

$$
\sup _{t} \int\left(k^{2}+1\right)\left|\breve{s}_{2}(t, k)\right|^{2} d \rho(E)<\infty
$$

since

$$
\left(H s_{2}, s_{2}\right)=\int_{0}^{\infty}\left(\left|s_{2}^{\prime}\right|^{2}+q\left|s_{2}\right|^{2}\right) d x
$$

Therefore,

$$
\limsup _{t \rightarrow \infty} \int_{M}^{\infty}|\breve{\psi}(t, k)|^{2} d \rho(E) \rightarrow 0
$$

as $M \rightarrow \infty$. We are left with proving

$$
\limsup _{t \rightarrow \infty}\left\|P_{(-\infty, \delta]} U(t) f\right\|_{2} \rightarrow 0
$$

as $\delta \rightarrow 0$. If $e_{j}(x)$ is eigenfunction for negative eigenvalue $-\kappa_{j}^{2}$ and $\left\|e_{j}\right\|_{L^{2}\left(\mathbb{R}^{+}\right)}=1$, then

$$
\left\langle U(t) f, e_{j}\right\rangle \rightarrow 0, \quad t \rightarrow \infty
$$

for each fixed $j$. Thus, we just have to show that $L^{2}$ norm of $U(t) f$ can not accumulate near zero energy, e.g., that

$$
\limsup _{t \rightarrow \infty}\left\|P_{[-\delta, \delta]} U(t) f\right\|_{2} \rightarrow 0, \quad \delta \rightarrow 0
$$

Write $q(x) \chi_{x>0}=q_{1}(x)+q_{2}(x)$, where

$$
\hat{q}_{1}(\omega)=\hat{q}(\omega) \chi_{|\omega|<1}, \quad q_{2}=q-q_{1}
$$

Clearly, $q_{1} \in W^{\infty, 2}\left(\mathbb{R}^{+}\right)$and $q_{2}=v^{\prime}$ where $v \in W^{1,2}(\mathbb{R})$. The multiplicative correction in $U(t)$ is

$$
\exp \left(-i \frac{t}{x} \int_{0}^{x} q(s) d s\right)=\exp \left(-i \frac{t}{x} \int_{0}^{x} q_{1}(s) d s\right) \exp \left(-i \frac{t}{x}(v(x)-v(0))\right)
$$

We have

$$
\left(\exp \left(-i t x^{-1} v(x)\right)-1\right) \frac{e^{i x^{2} /(4 t)}}{t^{1 / 2}} \hat{f}_{o}(x /(2 t)) \rightarrow 0
$$


in $L^{2}(\mathbb{R})$ as $t \rightarrow \infty$ since $\lim _{x \rightarrow \infty} v(x)=0$. Next, write

$$
\begin{aligned}
\exp \left(-i \frac{t}{x}\left(C+\int_{0}^{x} q_{1}(s) d s\right)\right) \frac{e^{i x^{2} /(4 t)}}{\sqrt{t}} \hat{f}_{o}(x /(2 t)) \\
\sim C_{1} \exp \left(-i \frac{t}{x}\left(C+\int_{0}^{x} q_{1}(s) d s\right)\right) \mathcal{F}\left(e^{-i t w^{2}} \hat{f}_{o}(\omega)\right)
\end{aligned}
$$

where $\mathcal{F}$ is the Fourier transform. Since $\hat{f}_{o}$ has support away from the zero,

$$
\mathcal{F}\left(e^{-i t w^{2}} \hat{f}_{o}(\omega)\right)=\psi^{\prime \prime}, \quad \psi=-\mathcal{F}\left(w^{-2} e^{-i t w^{2}} \hat{f}_{o}(\omega)\right)
$$

and $\psi$ travels ballistically in time as well. Therefore, we can write

$$
\exp \left(-i \frac{t}{x}\left(C+\int_{0}^{x} q_{1}(s) d s\right)\right) \mathcal{F}\left(e^{-i t w^{2}} \hat{f}_{o}(\omega)\right)=s_{1}^{\prime \prime}+s_{2}, \quad x>1
$$

where

$$
s_{1}=\exp \left(-i \frac{t}{x}\left(C+\int_{0}^{x} q_{1}(s) d s\right)\right) \psi
$$

Since $s_{1}$ travels ballistically, $\sup _{t}\left\|s_{1}\right\|_{W^{2,2}(\mathbb{R})}<\infty$, and $\left\|s_{2}\right\|_{2} \rightarrow 0$ as $t \rightarrow \infty$, we can write

$$
\exp \left(-i \frac{t}{x}\left(C+\int_{0}^{x} q_{1}(s) d s\right)\right) \mathcal{F}\left(e^{-i t w^{2}} \hat{f}_{o}(\omega)\right)=H l_{1}+l_{2}
$$

where $l_{1}(0)=0, \sup _{t}\left\|l_{1}\right\|_{W^{2,2}\left(\mathbb{R}^{+}\right)}<\infty,\left\|l_{2}\right\|_{2} \rightarrow \infty, \quad t \rightarrow \infty$. These representations show that

$$
\limsup _{t \rightarrow \infty} \int_{\delta}^{\delta}|\breve{\psi}(t, k)|^{2} d \rho(E) \rightarrow 0
$$

as $\delta \rightarrow 0$ (i.e. (0.8) holds). So,

$$
\frac{1}{T} \int_{0}^{T} d t \int_{\mathbb{R}}\left|\breve{\psi}(t, k)-\breve{W}_{f}(E)\right|^{2} d \rho(E) \rightarrow 0
$$

and the theorem is proved.

It is an interesting problem to try to relax $(0.1)$ to just $q \in L^{2}\left(\mathbb{R}^{+}\right)$. We are not able to do that at this moment.

\section{Acknowledgements}

This research was supported by Alfred P. Sloan Research Fellowship and NSF Grant DMS-0758239. 


\section{References}

[1] M. Christ, A. Kiselev. Scattering and wave operators for one-dimensional Schrödinger operators with slowly decaying nonsmooth potentials. Geom. Funct. Anal., 12 (2002), 1174-1234.

[2] S. Denisov, Wave equation with slowly decaying potential: asymptotics of solution and wave operators. preprint. 